\title{
A New Paradigm for SpeckNets: Inspiration from Fungal Colonies
}

\author{
Ruth Falconer, James Bown \\ SIMBIOS Centre, University of Abertay Dundee \\ Jon Timmis \\ University of York
}

Napier University, Edinburgh

\begin{abstract}
In this position paper, we propose the development of a new biologically inspired paradigm based on fungal colonies, for the application to pervasive adaptive systems. Fungal colonies have a number of properties that make them an excellent candidate for inspiration for engineered systems. Here we propose the application of such inspiration to a speckled computing platform. We argue that properties from fungal colonies map well to properties and requirements for controlling SpeckNets and suggest that an existing mathematical model of a fungal colony can developed into a new computational paradigm.
\end{abstract}

\section{Introduction}

As access to information becomes universal, with vast quantities of information stored transparently across multiple, distributed sources through ubiquitous computational devices, a paradigm shift in the way that systems are developed and managed is required. Current methodologies in both software and hardware engineering will not suffice when attempting to develop networks that are selfmanaging, self-diagnostic, and above all, robust and secure in ever changing dynamic and unpredictable environments. The complexity of such systems goes beyond the capabilities of traditional computer science and software engineering abstractions [10] — yet advancements in micro-electromechanical systems technology, wireless communications, and digital electronics have already enabled the development of exceptionally small mechanical devices that are inexpensive, low-power and sense phenomena in the physical world that can be connected together in large numbers to form wireless sensor networks. An example of such as device is a Speck; a minute device which is envisaged to open up a new generation of "spray-on computers" [9], in which dense networks, SpeckNets, consisting of thousands of nodes can be created. However, although advances in technology allow such devices to be physically created, the software required to enable these devices to function in a useful manner lags far behind.

The hurdles that need to be overcome in order for Specknets or similar networks of pervasive devices to even function appear unsurmountable when considered in engineering terms, yet many natural systems deal highly successfully with such problems on a continuous basis. For example, the central nervous system and the immune system function both function highly successfully in an autonomic manner, and are decentralised, scalable, robust, and require low-maintenance costs - items on the wish-list of any software-engineer. The computational world is increasingly looking to the biological world for solutions, e.g see [5] for a review which covers for example ant-colonies, swarm systems and molecular systems. The goal of this paper however is to introduce a further natural paradigm, that of fungal networks. To date, this natural system has not been properly explored as a metaphor in any computational context but offers fascinating possibilities for developing a new paradigm for working with pervasive systems.

Fungi are thought to represent the largest and oldest organisms on Earth. There are an estimated 1.5 million species of fungi in the world, and they are so abundant that they constitute an entire kingdom of Life. In spite of their abundance, they are largely invisible since they mostly inhabit an opaque substrate (soil); indeed, only 70,000 species are known. Fungal growth, often referred to as the mycelium, allows for the potentially indefinite existence of a colony ${ }^{1}$. This growth form is indeterminate and unlike most determinate organisms fungi appear to have no programmed upper limits in its spatial and temporal extent. These colonies exhibit precisely the properties sought after in pervasive systems; emergence and decentralisation; plasticity and remodelling; resilience and robustness; invasion prevention and self-healing; self-organisation, optimisation and complexity.

\footnotetext{
${ }^{1} \mathrm{~A}$ single colony is growing in the subterranean world of a Michigan forest, and its spatial extent, weight and age are approximated to be 15 hectares, 100 tonnes and 1,500 years respectively
} 


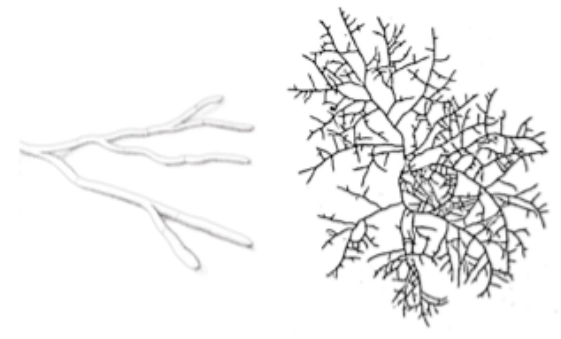

Figure 1. (a) Hyphae and associated branching (b) An integrated hyphal network (mycelium) growing on a homogeneous resource. As can be seen the network has a complex topology that characterises fungal colony growth. This topology may become asymmetric for heterogeneous environments.

In the next section, we give a brief introduction to the biological properties of fungal systems, emphasising the properties which are relevant to pervasive systems. This is followed by a more detailed description of Specknets (as an example of Pervasive Adaptive system in which we have already applied other biological paradigms), in which we elucidate the high-level mapping between the properties required in a SpeckNet and that found in fungal systems.

\section{Fungal Colonies}

A typical fungal colony comprises a vegetative mycelium and a range of differentiated structures such as reproductive organs (the mushrooms that are commonly seen beside trees in the forest in Autumn) and survival or resting structures (e.g., storage structures termed scelerotia). The colony is made up of a complex network of interconnected and interacting hyphae, tubes that have diameters ranging between one and thirty micrometers (figure 1). The colony undergoes four main processes for maintenance of the mycelium: uptake, redistribution, recycling and growth. Fungi uptake resource from the environment at the tips of the mycelium, and grow by converting that uptake into apical extension, i.e., formation of the tip. This creates a filamentous growth form that allows a pervasive and efficient exploitation of spatially heterogeneous environments. Fungi are sophisticated, dynamic transport agents, and acquired nutrients are typically redistributed within this hyphal network to areas requiring investment. Investment can be in the form of apical extension (i.e., growth of the mycelium where external resource is high), production of reproductive fruiting bodies and scelerotia, and the produc- tion of enzymes and inhibitor compounds to ward off competitors.

This transport of nutrients within the colony allows a form of non-local communication among different parts of the individual. This communication can be enhanced by some hyphae fusing and becoming anastomosised (see centre of figure 1(b)). Thus the colony can be considered an integrated unit and is more than the sum of its individual hyphae [3]. Different events occurring within the colony cannot therefore be totally independent and the local environments occurring within the mycelial boundary cannot all be treated as entirely separate domains. Fungi are constantly responding to the environment by both reacting to and governing nutrient flow within the hyphal network. In essence the colony structure remodels itself as a consequence of nutrient discovery, destructive disturbance and biotic and abiotic factors. As well as investing in areas of high metabolic activity, fungal colonies are also effective at divestment where necessary. Aged parts of the mycelium are often recycled (mobilised) and emptied of protoplasmic contents. This recycled internal resource and biomass may be transported to younger parts of the colony or areas requiring other forms of investment (e.g. antibiotic production). This recycling allows the colony to essentially reallocate itself (figure 2), degrading in the areas that have become redundant and growing into unexplored territory in search of new resources to exploit. By effectively routing nutrients and mobilised (recycled) biomass to the areas of the colony in most need, fungi respond rapidly to changes in environmental context. The mycelium may therefore be thought of as an interconnected, cooperative network where the local environment of one part may affect distant parts [7]. Growth of the network requires a positive feedback, resulting from the uptake of resource from the environment leading to an increase in biomass that subsequently increases uptake and is thus autocatalytic resulting in an expansive drive. Further, negative feedback, determined by a low concentration of nutrients and the transport mechanisms involved in the transport of nutrients within the network, counters the expansive drive of the mycelium.

\subsection{Fungal colony properties}

The properties of fungal colonies that are potentially of interest to the design of pervasive adaptive systems are now discussed:

1. Emergence and decentralisation Patterns produced are a consequence of interactions between many individual parts, i.e., hyphae, making up the system. There exists a set of biological processes operating at a local level that determine the growth characteristic of individual elements that in turn depend on local context. A global pattern emerges from these local interactions 


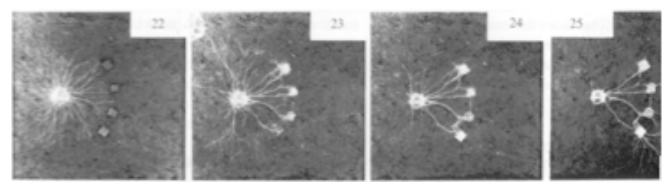

Figure 2. The fungal colony growing from the white resource on left hand side of diagram optimises its topology in response to nutrient discover to maximise the amount of resource obtained from the environment. Investment where nutrients are discovered and divestment where there is a lack of resource, reproduced from [1]

and there is no centralised control responsible for the pattern that emerges.

2. Plasticity and remodelling Fungi are dynamic and versatile organisms capable of changing their form as a result of the environmental conditions. They appear to self-regulate by altering their internal partitioning of resource and are thus capable of varying the balance between exploration and assimilation, conservation and redistribution of resources in response to the local environment [8]. They have evolved efficient mechanisms for routing resource throughout the network structure as a whole. This routing is based on factors such as exploitation capacity, invasion prevention and self-healing and it is via the partitioning and transport that the topology of the fungal network emerges.

3. Resilience and Robustness Fungi resist perturbation and stress by automatically creating differentiated structures within the colony form. Scelerotia, i.e., storage structures, enable fungi to survive when resource is low. These structures store resource within a thick, melanised outer structure. Mycelial cords, also termed mycelial strands, consist of linear aggregations of hyphae. These enable rapid relocation of resource and develop in response to environmental stresses and, in their mature regions, are composed of a wide impermeable cell wall. Relocation of resource in fungal networks is robust as they can have many connected pathways, for example, an Armillaria lutea network posses 5.6 E 21 minimal spanning trees. Further, parts of the network can shut down, by closing off the hyphae, stopping resource flow to and from the area undergoing stress or perturbation. Fungal resilience is also exemplified in the switching from exploitative, phalangeal growth form to an explorative guerilla-strategy, an escapist response to grazing insects [2].
4. Invasion Prevention and Self Healing Fungi adapt quickly and effectively when lines of communication become severed, blocked or broken by sealing off the area and re-routing resources via an alternative path. If the damage caused by disruption is not too severe resources may also be reallocated to the area of damage in order to rebuild the cell wall material. Fungi have sophisticated anti invasion mechanism, and an example of fungal invasion is combative fungal interactions. The mechanisms of combative fungal interactions include antagonism at a distance, hyphal interference, mycoparasitism and gross mycelial contact. These result in intermingling of species, deadlock, engulfment or replacement and others. Antagonism at a distance involves the release of a diffusible or volatile compound [2] that inhibits the growth of a fungal species. This can result in the replacement of one species by the other or mutual inhibition resulting in deadlock. Deadlock is often an outcome of fungal interactions on agar. Hyphal interference occurs when non-self hyphae are in close proximity and is mediated through a diffusible metabolite. Upon detection of non-self hyphae, the hyphae of one or both colonies may degenerate often referred to as lysis. Mycoparasitism occurs upon contact and recognition of a host, subsequently growth over the host occurs from which nutrients can be obtained biotrophically or necrotrophically [2]. Gross mycelial contact occurs when both fungi grow into one another and is often followed by a change in colony morphologies resulting in the production of differentiated structures such as barrages and sclerotia; pigmentation changes and redistribution of the mycelium can also occur. In some cases if the hyphae of two fungal individuals are somatically compatible, usually self pairings, intermingling of hyphae will occur forming one dense mycelial mat and this is a neutralistic interaction.

5. Self-organisation, optimisation and complexity A switch from exploitative to explorative behaviour exemplifies self-organisation and self-optimisation in fungal colonies. For example, rapid exploitative growth when hyphae contact a new food supply is effected by mobilizing and reallocating resources to exploit the new food sources, and explorative growth is observed when nutrients are scarce. The coordinated and decentralised effort of the colony in building differentiated structures is also a form of self-organisation. Fungal networks can be described as a complex system because cooperation, selforganisation, emergence and non-linearities in colony dynamics are key to the fungal form. Cooperation is mediated through the transport of internal resources allowing distal regions to communicate. Finally, as with 


\begin{tabular}{||c|c||}
\hline Telecommunications Network & Fungal Network \\
\hline Data packets & External Resource \\
Traffic & Mobile biomass \\
Nodes & Immobile biomass \\
Bandwidth & uptake \\
Energy capacity & Immobile biomass \\
Traffic density & Mobile biomass transfer \\
Route reinforcement & Biomass recycling \\
\hline
\end{tabular}

Table 1. An explicit mapping between a telecommnications routing network and a fungal colony

the vast majority of complex systems encountered in nature, non-linearities often characterise regulatory interactions, i.e., switching a process on or off depending on the signal and will play an important role in the development of fungal networks [6]. In fungi this may be the regulatory mechanisms such as nutrient repression controlling the biosynthesis of secondary metabolites.

Given these properties, initial investigations (not reported here) have already been undertaken to investigate the applicability of fungal networks to routing traffic in a telecommunications system using a mapping shown in table 1. This mapping was used to design an algorithm to perform routing in a decentralised network trafficking system, which is able to respond to dynamic loading. The algorithm provided promising results when compared to an ant-colony routing algorithm. Encouraged by these results, we now describe how a similar mapping can be made to a SpeckNet environment.

\section{SpeckNets}

We now describe the properties of the subclass of wireless sensor networks known as SpeckNets which we suggest has properties which naturally map to the fungal network paradigm. Although inspiration from the natural immune system has already been used in the design of algorithms for SpeckNets [4], to date, no other biological inspiration has been applied in this domain.

The first generation of Specks, which are currently in existence, have a dimension of approximately five millimetres, and are known as ProSpeckz (Programmable Specks over Zigbee Radio). A typical Speck comprises of a computeron-a-chip, that combines a micro-processor and memory (FLASH and RAM), a radio chipset,an antenna that allows communication ranges from a few centimetres to over a few meters, a power supply, such as compact rechargeable batteries, and a number of sensors, depending on the type of application. The minute dimensions of a Speck affects all constituent parts of a speck node, and consequently, the overall performance and efficiency of SpeckNets. The small physical dimensions imply limited space for loading on board memory and power supply, and as a result, storage capacity of specks is extremely limited (usually less than $10 \mathrm{~Kb}$ of FLASH memory and $1 \mathrm{~Kb}$ at most of RAM). Processing power is also restricted because of the above constraints, and especially due to the memory constraints. Sensors carried by each speck perform data capture, while inbuilt processing capabilities permit specks to filter data and extract information from the environment and LED components provide specks with a feedback mechanism. Clearly, Specks impose both considerable physical and software engineering challenges; we propose a mapping to the properties of fungal colonies below which may represent a way forward:

1. Emergence and decentralisation One of the defining features of a Specknet (as opposed to traditional WSNs) is the lack of powerful base station units. This forces the network to operate in a decentralised and asynchronous fashion, by sharing tasks and processes between its autonomous units. Distributed functioning is enabled by incorporating communication capabilities into each individual Speck, which allow the constituent devices to interact with each other. Individual devices can be unreliable; the system as a whole must find its way to co-ordination through alternative pathways.

Interactions between the numerous autonomous, spatially distributed units in the network must result in coherent global behaviours. These behaviours generally cannot be pre-specified in exact terms, but must emerge from the network through interactions of their constituent elements only. Engineering such platforms using traditional object-oriented and component-based methodologies becomes inefficient [10]; new paradigms must be found which enable global behaviours to emerge in massive, decentralised networks.

In a fungal network, a set of biological processes operating a local level determines growth characteristics which depend on local context. In SpeckNet, data routing, which is analogous to the biological process of internal transport, will depend on local context such as the power capacity and current traffic density of connected SpeckNet neighbours. External local conditions, i.e. stimuli, will affect data gathering and traffic generation, i.e. response, e.g. a rise of temperature may trigger the speck to sample the environment more frequently.

2. Plasticity and remodelling Individual units in a 
SpeckNet may be programmed with a number of 'classes' of functionality. In response to changes in local areas of the network, individual specks may have to switch functionality depending on the current needs of the network. For example, this may occur in response to exogeneous changes; a sudden rise in temperature in the network might trigger a speck to sample the environment more frequently or on the other hand, may cause the speck to switch from monitor the environment to broadcasting warning messages in its local area. On the other hand, a speck may need to modifiy its behaviour due to changes in endogenous conditions, for example in order to conserve battery power or due to failure of other internal components. Therefore, it is essential that both single specks and the entire network are plastic in their behaviours and responses.

A key aspect of this plasticity is the ability to (re)distribute work load in the network, depending on the current state of the network. Strategies observed in fungal networks provide some inspiration for this: through biomass recycling, fungal networks are able to reconfigure their architecture to both exploit new external resource through increased allocation and to reduce biomass allocation in areas that no longer provide resource. Where external resource is a correlate of workload requirements for SpeckNets, the Speck network may switch on and off regions in response to localised workload changes in line with increased allocation/ reduction in workload processing as required.

3. Resilience and Robustness The physical characteristics of speck devices inevitably render them unreliable in many respects. Wireless communication is not only unreliable but is of limited range. For example, to minimise the size of the antenna $(13 \times 6 \mathrm{~mm})$, operation is set at high frequencies $(2.4 \mathrm{GHz})$ - this however incurs high path losses. The most crucial constraint in SpeckNets is to minimise power consumption on every design level. As noted above, speck "death" due to loss of power or otherwise can result in nodes permanently being removed from the network (in terms of functioning). At other times, Specks may periodically switch to "sleep mode", during which they are unavailable for participation in the network. Other, unpredictable node failures can occur for a number of reasons - the specks may even be placed in open and sometimes harsh, environments (for example in sewers). All of these factors contribute to an unpredictable effect on the functioning of the speck network, yet the network must remain resilient and robust to these changes, maintaining global functionality at all times. This requirement has a direct analogy to the ability of fungal colonies to resist perturbation and stress by au- tomatically creating differentiated structures within the colony form.

Specifically, it may be possible to have different type of signals or warning messages associated with data packets that can potentially shut down parts of the network. The relocation and finding of alternative routes would emerge in response to the change in traffic density resulting from shutting down areas of the network, just as fungi recycle biomass in older, less active parts and reallocate biomass to other more active regions of the network.

4. Invasion Prevention and Self Healing Just as in a fungal colony, where lines of communication can become severed or broken, local regions within a SpeckNet may become cut-off or unreachable due to malfunctioning devices (which may be terminally unrepairable or simply sleeping or needing recharging). In such circumstances, messages need to be re-routed to avoid 'dead' areas of the network; the Specknet must be able to deal with these circumstances. In a fungal network, cells can physically rebuild cell walls; although technology does not yet extend to analagous processes in Specks (e.g recharging via another Speck) it is possible for virtual rebuilding to take place in the network where Specks switch to an alternative type of behaviour or functionality to address deficiencies in the network. Potential methodologies for achieving this in a Specknet are discussed in [4].

5. Self-organisation, optimisation and complexity Specknets embrace the idea of an autonomous system with programmable constituent parts, where the parts themselves are sustaining the system. A SpeckNet is clearly an example of a complex system, in which individually weak, spatially distributed devices interact to produce complex, emergent behaviours. The system must exhibit 'self' properties such as self-organisation, self-sufficiency and self-adaptation. As in the fungal colonies, these global, collective behaviours cannot simply be mapped from the individual behaviours; the SpeckNet must exhibit non-linearity in that its global behaviour is greater than the sum of its constituent parts. The complexity of the SpeckNet - as in a fungal colony - arises not only due to its necessity to selforganise but also in its need to continuously adapt to its own information processing and to its environment.

Further, the challenging and unusual hardware of a Speck device imposes a considerable requirement for optimisation within a Specknet. Technically, the most expensive activity in small sensor nodes is radio usage. Data transmission and reception usually draw significant amounts of power, in comparison with processor 
and sensors [9], for example, sending a single bit can consume the same energy as executing 1000 instructions. Energy drain leads to speck "death", which is also exacerbated by the placement in open environments, where it is not always possible to fully control operating conditions. Thus, Specks cannot simply broadcast at will. Rather the network as a whole must self-organise in a manner in which efficient routing and of messages can occur in order to transmit essential information through that network, and react accordingly.

A fungal colony has been shown to exhibit both $e x-$ ploitative and exploratory capabilities which are crucial concepts when discussing self-organisation. Mapping this to SpeckNets, we can consider an example of an exploitative behaviours as self-organising so as to route traffic through nodes with high power or battery capability, while the corresponding explorative behaviour might be thought of as minimal routing through routes with lower power capacity, until a higher capacity route can be found. Clearly, other such behaviours might be realised depending on the particular application of the SpeckNet.

\section{Realising a Fungal Colony Algorithm}

In the previous section we outlined at a motivational level a proposed mapping between SpeckNets and Fungal Colonies. A mathematical model of fungal growth and interactions (based on reaction-diffusion systems and solved numerically) has already been proposed by Falconer $e t$ al in [6]. This will be used to inform and abstract theoretical concepts that may be useful for SpeckNets (and was previously used tto implement the telecommunications network mentioned in section 2. The details of the model are outwith the scope of this paper but can be found in full in [6]; essentially the model represents an individual mycelial network as biomass comprising of three fractions: immobilized insulated biomass $b_{i}$, non-insulated biomass $b_{n}$ and mobilized biomass $n$ and models them via continuous density distributions. As a SpeckNet is a discrete network consisting of nodes and edges and not a continuous density distribution as in [6] the fungal model will be discretised in terms of hyphae which will represent edges and hyphal branches/intersections will represent nodes. The new model will be likely to be based on cellular automata and agentbased modelling paradigms.

\section{Conclusion}

Biological systems have provided inspiration over many years for solving a wide variety of problems. In this paper, we have explored the potential use of novel biological paradigm, that of fungal colonies. It is expected that this mapping will be instructive in translating fungal networks to SpeckNets. for the inspiration in low-cost, low-power wireless sensor networks, specifically speckled computing based networks: SpeckNets

Properties such as decentralisation, emergence, plasticity, resilience, robustness and self-star that are inherent to fungal colonies map well to desirable properties in SpeckNets. There are many challenges to building SpeckNet devices, and a low-cost, low-power solution is required. Given the natural mapping between fungal colonies and SpeckNets, we suggest it is a worthy endeavor to investigate further the potential exploitation of fungal colonies in the context of pervasive adaptive systems.

\section{References}

[1] L. Boddy. Saprotrophic cord-forming fungi: meeting the challenge of heterogeneous environments. Mycologia, 91:13-32, 1999.

[2] L. Boddy. Interspecific combative interactions between wood-decaying basidiomycetes. FEMS Microbiology Ecology, 31:185-194, 2000.

[3] J. L. Bown, C. Sturrock, W. Samson, Staines, H.J., J. Palfreyman, K. White, N.A.and Ritz, and J. Crawford. Evidence for emergent behaviour in the community-scale dynamics of a fungal microcosm. Proc. R. Soc. Lond. B, 266:1947-1952, 1999.

[4] D. Davoudani, E. Hart, and B. Paechter. Computing the state of specknets: Further analysis of an innate immuneinspired model. In Artificial Immune Systems: Proceedings of ICARIS 2008, 2008.

[5] F. Dressler. Benefits of bio-inspired technologies for networked embedded systems: An overview. In Organic Computing - Controlled Emergence, 2006.

[6] R. Falconer, J. Bown, N. White, and J. Crawford. Biomass recycling and the origin of phenotype in fungal mycelia. Proc. Roy. Soc B. Lond, 272:1727-1734, 2005.

[7] S. Lindahl and S. Olsson. Fungal translocation and creating and responding to environmental heterogeneity. Mycologist, 18:79-88, 2004.

[8] A. Rayner, G. Griffith, and A. Ainsworth. Mycelial interconnectedness. in the growing fungus. London: Chapman and Hall, 1995.

[9] K. Wong and D. Arvind. Speckled computing: Disruptive technology for networked information appliances. In Proceedings of the IEEE International Symposium on Consumer Electronics (ISCE'04) (UK), pages 219-223, September 2004.

[10] F. Zambonelli and H. V. D. Parunak. Signs of a Revolution in Computer Science and Software Engineering. In Engineering Societies in the Agents World III: Third Int. Workshop, ESAW 2002, Madrid, Spain, September 16-17, 2002. Revised Papers, pages 13-28, 2002. 